$\mathrm{e}^{+} \mathrm{e}^{-}$Collisions from Phi to Psi 2013 (PHIPSI2013)

International Journal of Modern Physics: Conference Series

Vol. 35 (2014) 1460423 (8 pages)

(C) The Authors

DOI: $10.1142 /$ S2010194514604232

\title{
THEORETICAL AND EXPERIMENTAL REVIEW ON PROTON FORM FACTORS
}

\author{
RINALDO BALDINI FERROLI \\ Laboratori Nazionali di Frascati dell'INFN, \\ Via E. Fermi 40, Frascati, 00044, Italy \\ rinaldo.baldini@centrofermi.it \\ SIMONE PACETTI \\ Dipartimento di Fisica e Geologia dell'Università degli Studi di Perugia, \\ Via A. Pascoli, Perugia, 06123, Italy \\ simone.pacetti@pg.infn.it
}

Published 18 December 2014

\begin{abstract}
During the last three lustra nucleon form factors experiments have lived a golden age, full of interesting results, that likely will continue and culminate when new data will come from BESIII, SND, CMD3 and PANDA, in the time-like region and, Jefferson Lab and A1 in the space-like region. On the other hand, from theoretical point of view, mainly concerning the possibility of descriptions in all kinematical regions, no great breakthrough has been made.
\end{abstract}

Keywords: Nucleon form factors.

PACS Numbers: 13.40.Gp, 13.40.Ks, 13.66.Bc, 13.85.Dz, 12.40.Vv

\section{Definitions and fundamental properties}

Electromagnetic (EM) form factors (FFs) of a hadron $h$ parametrize the nonpointlike nature of the vertex $h \gamma^{*} h$. Assuming gauge and Lorentz invariance, the EM current of a hadron of spin $S$ contains $2 S+1$ independent FFs; they are unknown Lorentz scalar functions of the squared momentum of the virtual photon. In case of nucleons, $S=1 / 2$, the EM current is written in terms of two FFs as

$$
\mathcal{J}^{\mu}=\bar{u}\left(p_{2}\right)\left[\gamma^{\mu} F_{1}\left(q^{2}\right)+\frac{i \sigma^{\mu \nu} q_{\nu}}{2 M} F_{2}\left(q^{2}\right)\right] u\left(p_{1}\right), \quad \sigma^{\mu \nu}=\frac{i}{2}\left[\gamma^{\mu}, \gamma^{\nu}\right],
$$

This is an Open Access article published by World Scientific Publishing Company. It is distributed under the terms of the Creative Commons Attribution 3.0 (CC-BY) License. Further distribution of this work is permitted, provided the original work is properly cited. 


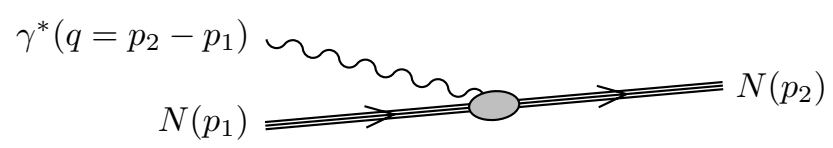

Fig. 1. Nucleon electromagnetic vertex, 4-momenta are reported in parentheses. The gray oval depicts the non-pointlike nature of the interaction and hence the FFs.

where 4-momenta follow the labelling of Fig. $1, M$ is the nucleon mass and, $F_{1}$ and $F_{2}$ are the Dirac and Pauli FFs. These FFs are not unique; each pair of linear independent combinations of $F_{1}$ and $F_{2}$ is equivalent, because defines unambiguously the structure of the vertex $N \gamma^{*} N$ ( $N$ stands for nucleon). Of particular interest are the so-called Sachs electric, $G_{E}$, and magnetic, $G_{M}$, FFs

$$
G_{E}\left(q^{2}\right)=F_{1}\left(q^{2}\right)+\tau F_{2}\left(q^{2}\right), \quad G_{M}\left(q^{2}\right)=F_{1}\left(q^{2}\right)+F_{2}\left(q^{2}\right), \quad \tau=q^{2} /\left(4 M^{2}\right) .
$$

In fact in the reference system, called Breit frame, where there is no energy exchange, i.e.: $q=(0, \vec{q})$ and $p_{1,2}=(E, \mp \vec{q} / 2)$, they acquire a clear physical meaning, being Fourier transforms of charge and magnetization distributions of the nucleon.

In light of this, at $q^{2}=0$, Sachs FFs are normalized to the electric charge, $Q_{N}$, and magnetic moment, $\mu_{N}$ of the nucleon, i.e.: $G_{E}(0)=Q_{N}, G_{M}(0)=\mu_{N}$.

Physical FFs are assumed to be limit values, for real $q^{2}$, of more general functions analytic in the whole $q^{2}$-complex plane ${ }^{\mathrm{a}}$. Moreover, unitarity reduces the analyticity domain by introducing the cut $\left(4 m_{\pi}^{2}, \infty\right)$ on the positive real axis, due to all the allowed hadronic intermediate states that couple to the virtual photon. The threshold $q_{\mathrm{th}}=2 m_{\pi}$ is to the mass of the lightest intermediate state. Hermiticity of the current operator implies real FFs for $q^{2}<q_{\mathrm{th}}^{2}$, hence, for the Schwarz reflection principle, they have non-vanishing imaginary parts, on the edges of the time-like (TL) cut. Perturbative $\mathrm{QCD}^{2}$ constraints the space-like (SL) high$q^{2}$ behavior of FFs to follow well established power laws. Using the PhragménLindelöf theorem ${ }^{3}$ the same behavior can be extended also in the TL region and thus $G_{E, M}\left(q^{2}\right) \underset{\left|q^{2}\right| \rightarrow \infty}{\propto}\left(q^{2}\right)^{-2}$.

\section{Space-like proton data}

Nucleon FFs are extracted from differential and total cross section data for scattering, annihilation and, more recently, from polarization observables, in polarization transfer experiments. The differential cross section for the scattering $e^{-} N \rightarrow e^{-} N$, in Born approximation and in the laboratory frame (nucleon at rest), reads

$$
\frac{d \sigma}{d \Omega_{e}}=\frac{\alpha^{2}}{q^{2}}\left(\frac{\epsilon_{2}}{\epsilon_{1}}\right)^{2} \frac{\cos ^{2}\left(\frac{\theta_{e}}{2}\right)}{\sin ^{2}\left(\frac{\theta_{e}}{2}\right)}\left[2 \tau G_{M}^{2}\left(q^{2}\right) \tan ^{2}\left(\frac{\theta_{e}}{2}\right)-\frac{G_{E}^{2}\left(q^{2}\right)-\tau G_{M}^{2}\left(q^{2}\right)}{1-\tau}\right],
$$

\footnotetext{
${ }^{a}$ Even though analyticity of FFs can be proved at any order of perturbation theory, still there is no a rigorous general demonstration. ${ }^{1}$
} 

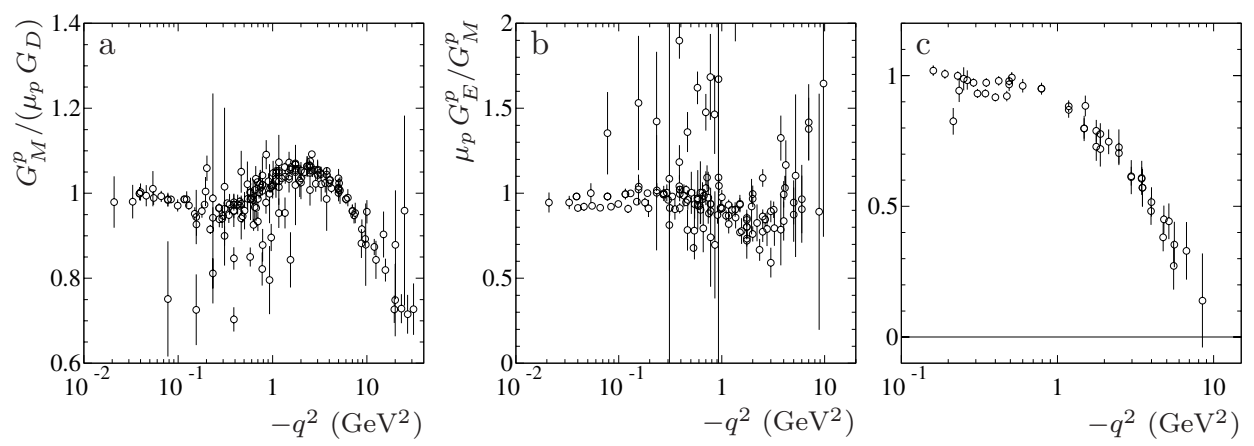

Fig. 2. World data ${ }^{5}$ on $G_{M}^{p} /\left(\mu_{p} G_{D}\right)$ (a). World data on $\mu_{p} G_{E}^{p} / G_{M}^{p}$ from unpolarized ep elastic scattering (b) ${ }^{5}$ and from polarization observables (c) ${ }^{6}$.

$\epsilon_{1(2)}$ and $\theta_{e}$ are the initial (final) energy and the scattering angle of the electron. The expression (3), known as Rosenbluth formula, ${ }^{4}$ allows to extract SL $\left(q^{2}<0\right)$ values of FFs at a given $q^{2}$, by comparing cross section measurements at different scattering angles. This procedure is called Rosenbluth separation technique. World collections of proton FF data, obtained using the Rosenbluth technique, are reported in Figs. 2a and 2b. In particular, Fig. 2a shows the magnetic proton SL FF normalized to its magnetic moment, $\mu_{p}$, and to the dipole $G_{D}=\left[1-\left(0.71 \mathrm{GeV}^{2} / q^{2}\right)\right]^{-2}$; while in Fig. $2 \mathrm{~b}$ is reported the ratio electric-to-normalized magnetic proton FF. These data suggest that, apart from the normalization at $q^{2}=0$, electric and magnetic proton FFs are both well described by the dipole behavior. From 1999, measurements of the ratio $G_{E}^{p} / G_{M}^{p}$ were performed with a new technique based on an idea due to A. I. Akhiezer and M. P. Rekalo, ${ }^{7}$ which consists in detecting the polarization transferred by polarized electrons on initially unpolarized proton targets. This procedure has the advantages of improving the accuracy and strongly reducing the dependence of the data on the radiative corrections.

\section{Time-like proton data}

Time-like FFs can be measured through the annihilation processes $e^{+} e^{-} \leftrightarrow p \bar{p}$. The cross section, for $p \bar{p}$ production (annihilation), in Born approximation and in the center of mass frame is

$$
\frac{d \sigma}{d \Omega}=\frac{\alpha^{2} \beta^{(-1)}}{4 q^{2}} \mathcal{C}\left[\left(1+\cos ^{2} \theta\right)\left|G_{M}^{p}\left(q^{2}\right)\right|^{2}+\frac{\sin ^{2} \theta}{\tau}\left|G_{E}^{p}\left(q^{2}\right)\right|^{2}\right], \quad \beta=\sqrt{1-\frac{1}{\tau}},
$$

where $\theta$ is the scattering angle, $\mathcal{C}$ is the Coulomb correction ${ }^{8}$ for the $p \bar{p}$ final (initial) state interaction and $\beta$ is the proton velocity. For $q^{2} \geq 4 M^{2}$, FFs are complex and only their moduli are measurable ${ }^{\mathrm{b}}$. However, the statistics available in the majority of the experiments is too low to perform precise studies of the angular distributions

\footnotetext{
${ }^{\mathrm{b}}$ Considering polarization also the phase of $G_{E} / G_{M}$ would be measurable.
} 

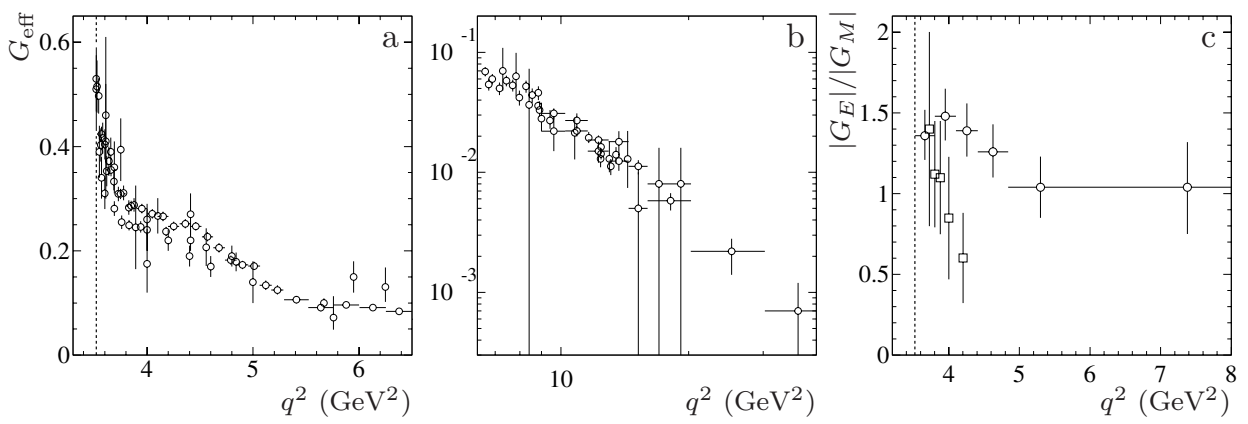

Fig. 3. World collection of data ${ }^{9}$ on effective proton FF, in the low (a) and high (b) momentum transfer regions. Vertical dashed lines indicate the physical threshold $q^{2}=4 M^{2}$. Ratio $\left|G_{E}\right| /\left|G_{M}\right|$ in the TL region. The data are from: Ref. [10] (empty squares) and Ref. [11] (empty circles).

needed to extract separate values of $\left|G_{M}^{p}\right|$ and $\left|G_{E}^{p}\right|$. Indeed, the quantity usually measured is an effective FF $G_{\text {eff }}$, corresponding to the common value of $\left|G_{E}^{p}\right|$ and $\left|G_{M}^{p}\right|$ if assumed equal each other. Figure 3 shows all the available data on $G_{\text {eff }}$. Only two experiments, PS170 ${ }^{10}$ and BaBar, ${ }^{11}$ have tried to extract TL moduli of proton electric and magnetic FFs separately. In both cases the measured quantity was $\left|G_{E}^{p}\right| /\left|G_{M}^{p}\right|$, even though PS170 ${ }^{10}$ and BaBar ${ }^{11}$ used two different processes: $p \bar{p} \rightarrow e^{+} e^{-}$and $e^{+} e^{-} \rightarrow p \bar{p} \gamma$, with initial state radiation technique. The two sets of data, shown in Fig. 3c, are incompatible. The PS170 measurement, that covers a small $q^{2}$-region close to threshold, agrees with: $\left|G_{E}^{p}\right|=\left|G_{M}^{p}\right|$, that holds only at the threshold ${ }^{\mathrm{c}} q^{2}=4 M^{2}$, see Eq. (2). BaBar data, which cover a wider energy range, verify, for the first time, the inequality $\left|G_{E}^{p}\right|>\left|G_{M}^{p}\right|$, up to $q^{2} \simeq 5 \mathrm{GeV}^{2}$.

\section{Nucleon form factors in theory and phenomenology}

Form factors represent the unknown elements of hadronic currents, being not computable in the framework of QED. Nevertheless some of their properties are strongly constrained by arguments based on first principles. In particular, FFs are required to be analytic functions of $q^{2}$ and hence we emphasize only those models which, by construction, can give descriptions valid in TL and SL regions.

\subsection{Vector meson dominance}

The earlier and most successful models used to parametrize nucleon FFs are based on the concept of vector meson dominance (VMD), i.e. the idea that the nucleonphoton interaction can be described in terms of intermediate vector mesons, with quantum numbers of the photon. Using VMD data can be well described in terms of few "physical" parameters as masses, widths and coupling constants. Massam and Zichichi were the first to conceive a VMD model to parametrize proton FFs in

${ }^{\mathrm{c}}$ Electric and magnetic FFs can be different at threshold if $F_{1}\left(4 M^{2}\right)$ and $F_{2}\left(4 M^{2}\right)$ are singular. 
both, SL and TL regions. ${ }^{12}$ In such a model, as in all the others, based on VMD, that followed, isospin components of the FFs were considered to well disentangle iso-scalar and iso-vector contributions. In 1972 Iachello, Jackson and Landé ${ }^{13}$ and in 1984 Gari and Krümpelmann ${ }^{14}$ proposed improved versions of the original model due to Massam and Zichichi. In particular, intrinsic FFs were introduced to reproduce the asymptotic behavior of pQCD. All these models provide good descriptions of available data of all nucleon FFs.

\subsection{Dispersion relation approaches}

Dispersion relations (DR) represent a powerful mathematical tool not only to handle FFs, but also to investigate their properties. Two kinds of DR approaches can be considered. The first uses VMD ${ }^{15}$ to construct TL imaginary parts of FFs, i.e. the spectral functions. Once such functions are known, usually in terms of vector meson masses, widths and coupling constants, DR are exploited to perform analytic continuations also in SL region. The second procedure defines quasi modelindependent analyses of data ${ }^{16}$ In this case DR are used both to check consistency of the data with theoretical constraints and to predict values of FFs at energies not experimentally accessible. The stability of these results depends on the accuracy of data, hence new precise measurements make this approach more and more reliable.

\subsection{Chiral perturbation theory}

Chiral perturbation theory $(\chi \mathrm{PT})$ is a low- $\left|q^{2}\right|$ effective filed theory of strong interaction. Below a typical energy scale $\Lambda \simeq 1 \mathrm{GeV}$ (with two flavors, $N_{f}=2$ ), the QCD Lagrangian is equivalent to that of $\chi \mathrm{PT}$, which depends on observable hadronic fields, only pions and nucleons in case of a leading-order description of nucleon FFs. Expressions of $F_{1,2}$, for instance, can be obtained considering tree and loop contributions, always in terms of pion and nucleon interactions. The free parameters of this model, which are constants accounting for the non-included heavier hadronic fields, are fitted to data. The low- $\left|q^{2}\right|$ limitation $\left(q^{2} \leq 0.4 \mathrm{GeV}^{2}\right.$ in Ref. [17]) does not allow to predict directly TL FFs, experimentally known only for $q^{2}>4 M^{2}$. Nevertheless, once the spectral functions are known, FFs can be reconstructed at any $q^{2}$, by means of DR. Using the optical theorem, spectral functions of nucleon FFs can be computed in $\chi \mathrm{PT}$ and moreover, the inclusion of vector meson contributions ${ }^{17}$ as well as the use of subtracted DR, helps in overcoming the problem of the low- $\left|q^{2}\right|$ restriction. However, so far, no analyses of such kind have been performed.

\subsection{Soliton models}

In soliton models, nucleons are represented by stationary topological solutions of a non-linear field theory, with only mesonic degrees of freedom, that approximates QCD in the limit of large number of colors ${ }^{18}$ In order to stabilize the solutions, further terms have to be added to the starting Lagrangian, which is that of the 
so-called non-linear $\sigma$ model. These terms describe vector mesons, that, with the peculiar repulsive nature of spin-1 particles, contrast the collapsing tendency of solitons.$^{19}$ The resulting Lagrangian is that of the so-called Skyrme model ${ }^{20}$ and its quantized soliton solutions are defined by radial functions, which represent the gauge fields. Space-like nucleon FFs are obtained as Fourier transforms of combinations of radial functions and their derivatives. The analytic continuation of these FF parametrizations can be obtained following the procedure of Ref. [21], that allows to well reproduce all the expected properties.

\subsection{Relativistic constituent quark models}

Hadrons and hence their FFs can be also microscopically described in terms of quark and gluon degrees of freedom, using constituent quark models. In case of nucleons, relativistic effects are important, and can be included in covariant way using the so-called light-front form..$^{22}$ A method to go beyond the "valence" approximation and then to include also non-valence components has been defined in Ref. [23] and it allows to extend the FF description from SL to TL region. The non-valence quarkantiquark-photon vertex is parametrized in terms of VMD. Even though a large number of resonances has to be considered to have a good description of data, the model has only few free parameters, because masses, widths, and coupling constants are either taken from data or computed following Ref. [24].

\subsection{Gauge-gravity correspondence}

The correspondence between gravity and string theory ${ }^{25}$ represents a promising possibility of finding analytic solutions of non-perturbative QCD. Such a correspondence is also called "holographic", since it connects spaces of different dimensions, a 5-dimensional anti-de Sitter $\left(\mathrm{AdS}_{5}\right)$ and a $N=4$ supersymmetric Yang-Mills gauge theory, which is a conformal field theories (CFT) in the typical $3+1$ Minkowskian space-time. In light of that, it could possible to compute observables of a stronglycoupled gauge theory in the framework of classical gravity. In general QCD is not conformal, however, in certain energy domains where quark masses are negligible, it can be approximated as a conformal theory, so that it corresponds to an $\mathrm{AdS}_{5}$. The fifth "holographic" coordinate $z$ can be interpreted as $z=1 / q$, the inverse of the probing momentum. Confinement is introduced geometrically by requiring $z \leq 1 / \Lambda_{\mathrm{QCD}} \cdot{ }^{26}$ Hadronic FFs can be defined in terms of Fourier transforms of $\mathrm{AdS}_{5}$ normalizable modes and external EM fields ${ }^{27}$ In case of nucleons, under suitable assumptions about the potential, ${ }^{27}$ the obtained SL FF expressions not only follow the power-law scaling predicted by perturbative QCD, but they have also a multipole structure, similar to that of VMD models. Such FF expressions have simple analytic forms and thus are well suitable to be continued also in the TL region, however, so far, no attempts have been done. 


\section{References}

1. S. Drell, F. Zachariasen, Proton electromagnetic form factors, 1st edn. (Oxford, 1961).

2. V. Matveev, R. Muradyan, A. Tavkhelidze, Teor. Mat. Fiz. 15, 332 (1973); S. J. Brodsky, G. R. Farrar, Phys. Rev. Lett. 31, 1153 (1973).

3. E. Titchmarsh, The Theory of Functions, (Oxford, 1939).

4. M. Rosenbluth, Phys. Rev. 79, 615 (1950).

5. C. F. Perdrisat, V. Punjabi, M. Vanderhaeghen, Prog. Part. Nucl. Phys. 59, 694 (2007).

6. M. Jones, et al., Phys. Rev. Lett. 84, 1398 (2000); V. Punjabi, et al., Phys. Rev. C 71, 055202 (2005); O. Gayou, et al., Phys. Rev. Lett. 88, 092301 (2002); A. Puckett, et al., Phys. Rev. Lett. 104, 242301 (2010); A. Puckett, et al., Phys. Rev. C 85, 045203 (2012); M. Jones, et al., Phys. Rev. C 74, 035201 (2006); G. Ron, et al., Phys. Rev. C 84, 055204 (2011); C. B. Crawford, et al., Phys. Rev. Lett. 98, 052301 (2007).

7. A. Akhiezer, M. Rekalo, Sov. Phys. Dokl. 13, 572 (1968); Sov. J. Part. Nucl. 4, 277 (1974).

8. A. Sommerfeld, Atombau und Spektralliniem, Vol. 2 (Vieweg, Braunschweig, 1944).

9. E. L. Lomon, S. Pacetti, Phys. Rev. D 85, 113004 (2012) [Err.-ibid. D 86, 039901 (2012)].

10. G. Bardin, et al., Nucl. Phys. B 411, 3 (1994).

11. J. Lees, et al., Phys. Rev. D 87, 092005 (2013).

12. T. Massam, A. Zichichi, Il Nuovo Cimento 43, 1137 (1966).

13. F. Iachello, A. Jackson, A. Landé, Phys. Lett. B 43, 191 (1973)l; F. Iachello, Q. Wan, Phys. Rev. C 69, 055204 (2004); R. Bijker, F. Iachello, Phys. Rev. C 69, 068201 (2004); Q. Wan, F. Iachello, Int. J. Mod. Phys. A 20, 1846 (2005).

14. M. Gari, W. Krümpelmann, Z. Phys. A 322, 689 (1985); Phys. Lett. B 173, 10 (1986); Phys. Lett. B 274, 159 (1992).

15. P. Mergell, U. G. Meissner, D. Drechsel, Nucl. Phys. A 596, 367 (1996); H. Hammer, U.-G. Meissner, D. Drechsel, Phys. Lett. B 385, 343 (1996); M. Belushkin, H.-W. Hammer, U.-G. Meissner, Phys. Rev. C 75, 035202 (2007).

16. B. Geshkenbein, B. Ioffe, M. A. Shifman, Yad. Fiz. 20, 128 (1974); S. Pacetti, Chin. Phys. C 34, 874 (2010); R. Baldini, et al., Eur. Phys. J. C 46, 421 (2006); R. Baldini, Eur. Phys. J. C 11, 709 (1999); S. Pacetti, Eur. Phys. J. A 32, 421 (2007); R. Baldini, et al., Nucl. Phys. A 755, 286 (2005).

17. B. Kubis,U.-G. Meissner, Nucl. Phys. A 679698 (2001).

18. E. Witten, Nucl. Phys. B 160, 57 (1979).

19. M. Bando, et al., Phys. Rev. Lett. 54, 1215 (1985); U. Meissner, N. Kaiser, W. Weise, Nucl. Phys. A, 466, 685 (1987).

20. T. Skyrme,Proc. Roy. Soc. Lond. A 260, 127 (1961).

21. S. Moretti, in these proceedings; A. Drago, S. Moretti, S. Pacetti, in preparation.

22. P. A. Dirac, Rev. Mod. Phys. 21, 392 (1949).

23. J. de Melo, it et al., Phys. Lett. B 671, 153 (2009); G. Salmé, Nucl. Phys. Proc. Suppl. 181-182, 51 (2008); J. de Melo, et al., Phys. Lett. B 581, 75 (2004); J. de Melo, et al., Phys. Rev. D 73, 074013 (2006).

24. T. Frederico, H.-C. Pauli, S.-G. Zhou, Phys. Rev. D 66, 054007 (2002); T. Frederico, H.-C. Pauli, S.-G. Zhou, Phys. Rev. D 66, 116011 (2002).

25. J. M. Maldacena, Adv. Theor. Math. Phys. 2, 231 (1998); S. Gubser, I. R. Klebanov, A. M. Polyakov, Phys. Lett. B 428, 105 (1998); E. Witten, Adv. Theor. Math. Phys. 2, 253 (1998).

26. J. Polchinski, M. J. Strassler, Phys. Rev. Lett. 88, 031601 (2002). 
27. J. Polchinski, M. J. Strassler, JHEP 0305, 012 (2003); S. J. Brodsky, G. F. de Teramond, Phys. Rev. D 77, 056007 (2008); A. Karch, E. Katz, D. T. Son, M. A. Stephanov, Phys. Rev. D 74, 015005 (2006); G. F. de Teramond, S. J. Brodsky, arXiv:1203.4025; S. J. Brodsky, G. F. de Teramond, Phys. Rev. Lett. 96, 201601 (2006). 